\title{
Oxytocin amplifies sex differences in human mate choice
}

5 Lei Xu, Benjamin Becker, Ruixue Luo, Xiaoxiao Zheng, Weihua Zhao, Qiong Zhang* and

6 Keith M. Kendrick ${ }^{*}$

8 The Clinical Hospital of Chengdu Brain Science, MOE Key Laboratory for

9 NeuroInformation, University of Electronic Science and Technology of China, Chengdu

10 611731, China.

11

$12{ }^{*}$ Corresponding authors:

13 Keith M. Kendrick

14 Address: No. 2006, Xiyuan Ave, West Hi-tech Zone, Chengdu 611731, China.

15 E-mail: kkendrick@uestc.edu.cn

16 Tel: 86-28-83201358.

17 Fax: 86-28-83201358.

18

19 Qiong Zhang

20 Address: No. 2006, Xiyuan Ave, West Hi-tech Zone, Chengdu 611731, China.

21 E-mail: zhangqiong320@uestc.edu.cn 


\section{Abstract}

25 Infidelity is the major cause of breakups and individuals with a history of infidelity are more

26 likely to repeat it, but may also present a greater opportunity for short-term sexual

27 relationships. Here in a pre-registered, double-blind study involving 160 subjects we report

28 that while both sexes valued faithful individuals most for long-term relationships, both single

29 men and those in a relationship were more interested in having short-term relationships with

30 previously unfaithful individuals than women. Oxytocin administration resulted in men rating

31 the faces of unfaithful women as more attractive but in women rating those of unfaithful men

32 as less attractive and also finding them less memorable. Oxytocin also increased men's

33 interest in having short-term relationships with previously unfaithful women whereas it

34 increased women's interest in having long-term relationships with faithful men. Thus,

35 oxytocin release during courtship may first act to amplify sex-dependent priorities in

36 attraction and mate choice before subsequently promoting romantic bonds.

38 Keywords: oxytocin, sex difference, mate choice, attraction, infidelity 


\section{Introduction}

41 Individuals who have previously been unfaithful in a relationship are over 3 times more likely

42 to repeat this in subsequent ones (Knopp et al., 2017), and infidelity is the most common

43 cause of divorce (Lansford, 2009). Infidelity in a partner represents a long-term relationship

44 risk to both sexes that can particularly impact negatively on females in terms of loss of

45 support for raising offspring but for males may also increase the risk of being cuckolded and

46 raising another male's offspring (Buss and Schmitt, 1993). Indeed, it is argued that this

47 difference in the perceived risk of infidelity by the sexes is reflected in women being more

48 concerned by emotional infidelity but men by sexual infidelity (Buss, 2018; Buss et al., 1992).

49 However, while both sexes clearly prefer fidelity in a prospective long-term partner men are

50 more likely to pursue short-term relationships and engage in casual sex in order to increase

51 their reproductive potential (Buss and Schmitt, 1993; Oliver and Hyde, 1993), although

52 women may do so to maximize their chance of reproducing with more masculine men who

53 have the highest levels of genetic fitness (Penton-Voak et al., 1999). There is also an element

54 of social learning in mate choice: "wanting women other men want or vice versa", known as

55 "mate-choice copying" (Place et al., 2010) which could be evidenced by knowledge that

56 individuals have had multiple affairs. As Scott Fitzgerald wrote of Gatsby's perception of

57 Daisy in "The Great Gatsby" (Fitzgerald, 1925): "It excited him, too, that many men had

58 already loved Daisy - it increased her value in his eyes". Overall therefore, individuals with a

59 previous history of infidelity could be considered as more attractive for short-term

60 relationships, due to a greater perceived potential availability for reproduction opportunities

61 and possibly greater genetic fitness. 
62 In terms of the biological underpinnings of evolutionary sex-differences in human mate

63 choice strategy, one potential candidate is the highly evolutionarily conserved neuropeptide

64 oxytocin (OXT) which plays a key role in the formation and maintenance of affiliative and

65 partner bonds in a number of species (Cavanaugh et al., 2014; Donaldson and Young, 2008;

66 Kendrick et al., 2017), including humans (Preckel et al., 2014; Scheele et al., 2012, 2013),

67 as well as in social learning (Hu et al., 2015) and conformity (De Dreu and Kret, 2016; R.

68 Luo et al., 2017). In humans, OXT facilitates sex-dependent differences in social priorities,

69 particularly in terms of positive or negative social attributes (Gao et al., 2016; L. Luo et al.,

70 2017; Scheele et al., 2014). Oxytocin can also sex-dependently facilitate approach or

71 avoidance behavior towards attractive strangers of the opposite sex although its effects can be

72 modulated by relationship status (Scheele et al., 2012). However, it is currently unknown

73 whether OXT may influence sex-differences in human mate-choice priorities.

74 Against this background we have therefore investigated whether sex-dependent biases in

75 patterns of mate choice revealed by knowledge of previous emotional or sexual

76 fidelity/infidelity in men and women who are either currently single or in a committed

77 relationship, are influenced by intranasal OXT administration. We hypothesized firstly that

78 under placebo (PLC) control conditions men would exhibit a preference for women who had

79 been unfaithful in a previous relationship whereas women would exhibit an aversion to

80 unfaithful men and instead prefer men who had previously been faithful. Secondly, we

81 hypothesized that under OXT such sex differences in mate preference would be enhanced and

82 particularly in single individuals who should have a greater priority for finding a partner than

83 those already in an established relationship. 


\section{Methods}

86 2.1 Participants. 160 heterosexual human subjects ( 80 males, age range 18-27 years) from

87 the University of Electronic Science and Technology of China (UESTC) were recruited to

88 take part in a double-blind, placebo-controlled, between-subject design experiment. An initial

89 power analysis showed that with this number of subjects the study had $80.7 \%$ statistical

90 power for detecting treatment and sex effects with a medium effect size of 0.45 (fpower.sas).

91 All subjects had normal or corrected-to-normal vision, were not color-blind and reported no

92 history of or current neurological or psychiatric disorders. Subjects were free of regular and

93 current use of medication and instructed to abstain from caffeine, nicotine and alcohol intake

94 the day before and on the day of the experiment. None of the female subjects was pregnant or

95 using oral contraceptives or tested at specific stages of their menstrual cycle. Using onset date

96 of previous menses and cycle length (mean \pm sem: $30.83 \pm 0.37$ days) provided by the

97 subjects we estimated (backward counting (Gangestad et al., 2016)) whether they were in

98 follicular phase (between the end of menses and ovulation, high conception risk) or luteal

99 phase (after ovulation and before the onset of menses, low conception risk) on the

100 experimental day (Penton-Voak et al., 1999). Eight females reported having irregular

101 menstrual cycles and were excluded for menstrual cycle related analysis. The proportion in

102 their follicular $(n=39 ; 22$ in the OXT group) or luteal ( $n=33 ; 16$ in the OXT group) phases

103 did not differ between the groups (Fisher's exact test: $p=0.636$, two-sided). There were no

104 significant menstrual cycle effects found for results obtained in the study itself (see SI). Both

105 subjects who were currently single ( $\mathrm{n}=82 ; 39$ males) and those who were currently in a 
106 committed relationship of $>6$ month duration $(32.00 \pm 2.45$ months; $\mathrm{n}=78 ; 41$ males $)$ were

107 included since relationship status can modulate OXT effects in men (Scheele et al., 2012;

108 Zhao et al., 2018). All single subjects were interested in finding a romantic partner and those

109 in a relationship reported that it was a stable exclusive one (indeed subjects in a relationship

110 scored significantly higher on the passionate love scale than single subjects $(102.09 \pm 1.55$ vs.

$11196.46 \pm 1.70-t(158)=2.442, p=0.016, d=0.387)$ providing further support for their being

112 in love). All subjects signed written informed consent and received monetary compensation

113 for their participation. The study was approved by the local ethics committee at the

114 University of Electronic Science and Technology of China and was in accordance with the

115 latest revision of the Declaration of Helsinki. The study was also pre-registered on the NIH

116 registration website $\quad$ (Trial $\quad$ ID: 2733237 ;

117 https://clinicaltrials.gov/ct2/show/NCT02733237).

118 To control for potential confounds, before intranasal treatment all subjects completed a

119 range of validated questionnaires (Chinese versions) measuring mood, personality traits and

120 attitudes toward love, trust and forgiveness (See SI). Univariate ANOVAs on questionnaires

121 and age showed no significant differences between the OXT- and PLC-treated males and

122 females (sex x treatment interaction: all $p s>0.070$; See Table 1).

1232.2 Intranasal administration. Subjects were randomly assigned to receive intranasal

124 administration of either OXT ( $n=80,40$ males and 40 females; 40 IU; OXT-Spray, Sichuan

125 Meike Pharmaceutical Co. Ltd, China; 5 puffs of 4 IU per nostril with a 30s interval between

126 each puff) or PLC ( $n=80,40$ males and 40 females; identical sprays with the same

127 ingredients other than the neuropeptide, i.e., glycerin and sodium chloride) following a 
128 standardized protocol (Guastella et al., 2013). In previous studies, we have found similar

129 behavioral and neural effects of 24 and 40 IU OXT doses, although the higher dose tended to

130 produce more consistent results (Geng et al., 2018; Xu et al., 2015; Zhao et al., 2017) and this

131 was recently supported by a study from another group showing dose-dependent effects using

132 these same doses (Shin et al., 2018). We therefore decided to use the higher 40 IU dose here

133 to try and maximize effects. Although we could not measure blood or cerebrospinal fluid

134 OXT concentrations following intranasal application other studies have reported that they

135 produce only relative small increases within the general physiological range (Quintana et al.,

136 2018; Striepens et al., 2013). While it is currently unclear whether intranasal OXT produces

137 direct effects on the brain or also indirectly via peripheral effects, it has been established that

138 OXT administered via this route does enter into the brain cerebroventricular system in

139 monkeys (Lee et al., 2018) and alters cerebral blood flow in an extensive number of brain

140 regions known to express OXT receptor mRNA in humans (Paloyelis et al., 2016). A recent

141 study comparing functional and brain effects of intranasal and intravenous OXT

142 administration also only found effects when it is given intranasally (Quintana et al., 2016).

143 For allocation of the participants to the two treatment groups a computer-generated list of

144 random numbers was used (groups, $\mathrm{n}=2$; numbers per group, $\mathrm{n}=40$; simple randomization).

145 Treatment allocation was done by an experimenter not involved in data acquisition and

146 analyses. Subjects and experimenter were blind to drug condition. In post experiment

147 interviews subjects were unable to guess better than chance whether they had received OXT

148 or PLC treatment (81 subjects guessed correctly; $\chi^{2}=0.025, \mathrm{p}=0.874$ ). In line with

149 standardized recommendations (Guastella et al., 2013) and two studies reporting 
150 pharmacodynamics of central effects of intranasal OXT in humans (Paloyelis et al., 2016;

151 Spengler et al., 2017) the experimental paradigm started 45 minutes after intranasal

152 treatment.

153 2.3 Stimuli. Before the formal experiment, we generated 54 sentences describing a behavior 154 indicative of fidelity or infidelity (either emotional or sexual; 12 14 sentences for each 155 behavior type) that a male or female individual had performed during a past relationship.

156 Sexual and emotional infidelity were defined as in Takahashi et al (Takahashi et al., 2006).

157 Sexual infidelity (or fidelity) included situations where a (or no) sexual relationship or deep

158 physical contact with other members of the opposite sex was indicated explicitly or implicitly.

159 Emotional infidelity (or fidelity) included situations indicating some (or no) form of romantic

160 emotional response or commitment to other members of the opposite sex. Each sentence was

161 written in Chinese, used the past tense and had male and female versions (i.e. "She......" for

162 male subjects in the study and "He....." for female subjects). In a pre-study, an independent

163 sample of forty volunteers (21 males) were asked to decide whether the behavior described

164 was an example of emotional or sexual infidelity/fidelity and also to rate how strong it was

165 using a 9-point scale. Based on the data from this pre-study, we selected 40 sentences (10 for

166 each behavior type) with a high discrimination between sexual and emotional fidelity or

167 infidelity (i.e. all the chosen sentences were correctly classified as representing fidelity or

168 infidelity behaviors by the raters and with a mean accuracy of $87.6 \%$ for distinguishing

169 emotional from sexual examples). There were no differences between male and female

170 examples in terms of discrimination accuracy or strength (all $p s>0.258$ ). Table S1 gives

171 examples of the emotional or sexual fidelity/infidelity behavior sentences. 
172 Facial images of 80 males and 80 females with neutral expressions were selected from an

173 in-house database of 260 face images following a pilot rating by 36 subjects (17 males) of

174 valence, attractiveness, likeability, trustworthiness of the faces from the opposite sex as well

175 as how aroused they were by them. All face images were carefully edited (removing

176 accessories or background details, but keeping hair, ears and neck) and presented in full color

177 at a 600×800 Pixel resolution on a black background (faces life-size). All selected faces were

178 rated as having a neutral valence (range 4.3-6.0; mean $=5.09$ ) and average attractiveness

179 (range 4.0-5.9, mean= 4.79), likeability (range 4.1-5.8, mean=4.81) and trustworthiness

180 (range 4.2-6.0, mean= 5.07). Half of the faces used for the rating task were divided randomly

181 into four groups (i.e. 10 faces per group for each sex). Mean valence, attractiveness,

182 likeability, trustworthiness and arousal ratings of the faces in each group did not differ

183 significantly for both male and female faces (ANOVAs all $p s>0.964$ ). Each group of faces

184 was assigned for pairing with sentences describing one of the four different fidelity/infidelity

185 types. Additionally, to control for possible face/sentence-group differences, the pairings of

186 face group and sentence type were randomized across individual subjects in the main study.

187 The remaining faces were used as novel stimuli in the recognition memory test and had

188 equivalent valence, attractiveness, likeability, trustworthiness and arousal ratings compared to

189 the faces paired with sentences for both sexes (all $p s>0.661$ ).

1902.4 Procedure. The experimental task (see Fig. 1) was presented on a computer with a

191 27-inch monitor (screen resolution: 1920*1080 pixels; refresh rate: $60 \mathrm{~Hz}$ ). In the rating task,

192 subjects viewed neutral expression face pictures of 40 unfamiliar members of the opposite

193 sex with average attractiveness paired with verbal information describing examples of how 
194 they had been either emotionally or sexually faithful or unfaithful during a previous

195 relationship (see Table S2). We included fidelity type as a factor since previous research has

196 reported that men are more influenced by sexual infidelity and women by emotional infidelity

197 (Buss, 2018; Buss et al., 1992). Subjects were told that these individuals were currently single

198 and instructed to view their faces, read the sentences describing their previous behavior

199 silently and then rate (on a 9-point scale) their facial attractiveness, likeability,

200 trustworthiness and arousal elicited by them based on their overall impression of them. Next,

201 subjects were asked whether they would like to have a short- or long-term romantic

202 relationship with the person (response options: "yes", "maybe" or "no" - see Fig.1). There

203 was no time limitation for subjects' responses. The percentage of "yes/maybe" responses

204 made by each subject for each condition indicated their willingness to have a relationship

205 with this kind of person.

206 Finally, subjects completed a surprise recognition memory test for these 40 faces

207 intermixed with another 40 novel faces (order of stimuli randomized). Each trial started with

208 a 600-800 ms fixation cross followed by a face presented for $1500 \mathrm{~ms}$ and subjects responded

209 whether the face was familiar or not without any time limitation. Four subjects had to be

210 excluded from this part of the analysis due to technical failures during data acquisition.

2112.5 Statistical Analysis. All data analyses were performed using SPSS 23.0 software (SPSS

212 Inc., Chicago, Illinois, USA). In all cases, data from rating scores, recognition memory

213 accuracy and percentage of "yes/maybe" responses for having a short- or long-term

214 relationship with a target individual were subjected to four (analysis of the PLC group alone)

215 or five (analysis of the PLC vs. OXT treatment groups) factor repeated-measures ANOVAs 
216 and significant $(p<0.05)$ main effects and relevant interactions were reported. Significant

217 interactions were explored using Simple Effect Tests, which were all Bonferroni-corrected

218 for multiple comparisons. For both ANOVAs and post-hoc tests measures of effect size are

219 given (Partial eta squared $\left(\eta_{\mathrm{p}}^{2}\right)$ or Cohen's $d$ ). Small, medium, and large effects were

220 represented respectively as $0.01,0.06$, and 0.14 for $\eta_{\mathrm{p}}^{2}, 0.20,0.50$, and 0.80 for Cohen's $d$

221 (Cohen, 1988).

\section{Results}

\section{$224 \quad 3.1$ Sex-differences on the impact of knowledge of previous fidelity or infidelity}

225 To identify treatment-independent sex differences on evaluations of a potential partner who

226 had previously displayed emotional or sexual fidelity or infidelity in a relationship, we first

227 analyzed data from the PLC control group using four-way repeated-measures ANOVAs with

228 fidelity (fidelity vs. infidelity) and type (emotional vs. sexual) as within-subject factors and

229 sex and relationship status as between-subject factors.

230 For attractiveness, likeability, trustworthiness and arousal ratings of the face pictures

231 paired with examples of fidelity or infidelity behaviors there were no significant fidelity $\mathrm{x}$ sex

232 interactions (all $p s>0.328$ ). However, there was a significant type $\mathrm{x}$ sex interaction for

233 likeability ratings $\left(F(1,76)=5.447, p=0.022, \eta_{\mathrm{p}}^{2}=0.067\right)$. Post hoc comparisons revealed

234 that women rated men who showed emotional fidelity or infidelity $(4.15 \pm 0.10,95 \% \mathrm{CI}=$

$235[3.96,4.34])$ more likeable than those who showed sexual fidelity or infidelity $(3.94 \pm 0.10$,

$23695 \% \mathrm{CI}=[3.74,4.15] ; p=0.001, d=0.366-$ see Fig. S1a). Thus, the most likeable potential

237 partners for men were those who showed sexual fidelity while for women they were those 
238 who showed emotional fidelity. There was also a similar trend for this with attractiveness

239 ratings although the interaction was only marginally significant $(F(1,76)=3.731, p=0.057$,

$240 \quad \eta_{\mathrm{p}}^{2}=0.047-$ see Fig. S1b). There were no significant interactions involving type and sex for

241 recognition memory accuracy (all $p s>0.094$ ).

242 For short-term relationship preferences, analysis in the PLC group revealed a significant

243 fidelity $\mathrm{x}$ sex interaction $\left(F(1,76)=8.807, p=0.004, \eta_{\mathrm{p}}^{2}=0.104\right)$. Post-hoc Bonferroni

244 corrected comparisons showed that $31.8 \pm 3.6 \%(95 \% \mathrm{CI}=[24.6 \%, 38.9 \%])$ of responses

245 made by men expressed interest (i.e. "yes" or "maybe" decisions) in having a short-term

246 relationship with an unfaithful individual, whereas only $17.0 \pm 3.6 \%(95 \% \mathrm{CI}=[9.8 \%$,

$24724.1 \%])$ of responses made by women $\operatorname{did}(p=0.005, d=0.658-$ see Fig. 2$)$. There were no

248 sex-differences for long-term relationship preferences (all $p s>0.109)$, with both men $(44.1 \pm$

$2494.9 \%, 95 \% \mathrm{CI}=[34.4 \%, 53.9 \%])$ and women $(47.9 \pm 4.9 \%, 95 \% \mathrm{CI}=[38.1 \%, 57.6 \%])$

250 showing an equivalent and greater preference for partners exhibiting previous fidelity $(p=$ $2510.589)$.

252 A separate analysis on female subjects found no evidence for a significant influence of

253 menstrual cycle stage (i.e. whether women were at a stage representing either a high or low

254 risk of conception) on any of the measures taken (see SI).

$255 \quad 3.2$ Effects of intranasal oxytocin on sex-differences in mate choice

256 To examine the effects of OXT on evaluations of potential partners showing previous fidelity

257 or infidelity, five way repeated-measures ANOVAs with fidelity (fidelity vs. infidelity) and 258 type (emotional vs. sexual) as within-subject factors and treatment, sex and relationship status 259 as between-subject factors were performed on rating scores, recognition memory accuracy 
260 and percentage of "yes/maybe" responses for having a short- or long-term relationship with a

261 target individual.

262 There were significant fidelity $\mathrm{x}$ treatment $\mathrm{x}$ sex interactions for attractiveness $(F(1,152)$

$263=8.454, p=0.004, \eta_{p}^{2}=0.053-$ see Fig. 3a) and likeability ratings $(F(1,152)=6.694, p=$

$2640.011, \eta_{\mathrm{p}}^{2}=0.042$ - see Fig. 3b). Post-hoc Bonferroni corrected comparisons showed that in

265 men OXT increased both face attractiveness $(p=0.047, d=0.421)$ and likeability $(p=0.017$,

$266 d=0.513$ ) of previously unfaithful women, while in women OXT decreased face

267 attractiveness $(p=0.016, d=0.529)$ and likeability $(p=0.181)$ of previously unfaithful men.

268 Thus, unlike the PLC group, in the group treated with OXT there were significant sex

269 differences in face attractiveness $(p<0.001, d=1.033)$ and likeability $(p<0.001, d=1.006)$

270 of previously unfaithful individuals. There were no significant OXT effects on face

271 attractiveness or likeability ratings given to previously faithful men and women (all $p s>$

272 0.461). And OXT did not alter the pattern of female subjects giving higher attractiveness or

273 likeability ratings than men for emotionally compared to sexually faithful individuals

274 (interactions involving type, treatment and sex: all $p s>0.260$ ). No significant interaction

275 effects involving treatment, sex, fidelity or type were found for trustworthiness (all $p s>$

2760.075 ) or arousal ratings (all $p s>0.134$ ) indicating that sex-dependent effects of OXT on

277 attraction ratings were specific.

278 Analysis of recognition memory accuracy for faces revealed a significant fidelity $\mathrm{x}$

279 treatment $\mathrm{x}$ sex interaction $\left(F(1,148)=6.036, p=0.015, \eta_{\mathrm{p}}^{2}=0.039\right.$; note: for this analysis 4

280 subjects were excluded due to incomplete data). Post-hoc Bonferroni corrected comparisons

281 demonstrated that women in the OXT group $(59.1 \pm 2.5 \%, 95 \% \mathrm{CI}=[54.3 \%, 64.0 \%])$ were 
282 less likely than women in the PLC group $(68.8 \pm 2.4 \%, 95 \% \mathrm{CI}=[63.9 \%, 73.6 \%])$ to

283 remember the faces of individuals who had previously exhibited infidelity $(p=0.006, d=$

2840.608 - see Fig. 3c). Oxytocin therefore effectively increased the chances that women would

285 only remember men with a history of being faithful. No other significant interaction effects

286 involving treatment, sex, fidelity or type were found (all $p s>0.243$ ).

287 For short-term relationship preference, analysis revealed a fidelity $\mathrm{x}$ treatment $\mathrm{x}$ sex $\mathrm{x}$

288 relationship status interaction $\left(F(1,152)=4.082, p=0.045, \eta_{\mathrm{p}}^{2}=0.026\right)$. Post-hoc Bonferroni

289 corrected comparisons showed that the percentage of yes/maybe responses given by single

290 men for having a short-term relationship with unfaithful women were increased from $30.0 \pm$

$2914.9 \%(95 \% \mathrm{CI}=[20.4 \%, 39.6 \%])$ in the PLC group to $45.8 \pm 5.0 \%(95 \% \mathrm{CI}=[35.9 \%$,

$29255.7 \%])$ in the OXT group $(p=0.025, d=0.643$ - see Fig. 4a).No other significant

293 interaction effects involving treatment, sex, fidelity or type were found (all $p s>0.06$ ).

294 For interest in having a long-term relationship there was a significant fidelity $\mathrm{x}$ treatment

$295 \mathrm{x}$ sex $\mathrm{x}$ relationship status interaction $\left(F(1,152)=5.439, p=0.021, \eta_{\mathrm{p}}^{2}=0.035\right)$. Post-hoc

296 Bonferroni corrected comparisons showed that the percentage of yes/maybe responses made

297 by single women for having a long-term relationship with faithful men were increased from

$29841.8 \pm 6.9 \%(95 \% \mathrm{CI}=[28.2 \%, 55.3 \%])$ in the PLC group to $64.1 \pm 6.4 \%(95 \% \mathrm{CI}=[51.5 \%$,

$29976.8 \%]$ ) in the OXT group ( $p=0.018, d=0.699-$ see Fig. 4b). No other significant

300 interaction effects involving treatment, sex, fidelity or type were found (all $p s>0.266$ ).

301 A separate analysis on female subjects found menstrual cycle stage did not influence the

302 OXT effects found above (see SI). 


\section{Discussion}

305 Overall, our findings demonstrate firstly that knowledge of previous fidelity and infidelity in

306 a prospective heterosexual partner effectively reveals sex differences in mate choice strategy.

307 Thus, men in the control PLC treated group generally exhibited greater interest in having a

308 short-term relationship with previously unfaithful individuals than women, and independent

309 of relationship status. There was no sex difference in the context of long-term relationships,

310 with both sexes showing an equivalent and greater preference for partners exhibiting previous

311 fidelity. Following OXT administration both men and women respectively exhibited

312 enhanced and reduced attraction to unfaithful individuals and women also found them less

313 memorable. Additionally, single men showed an increased preference for have a short-term

314 relationship with previously unfaithful women whereas single women showed an increased

315 preference for having a long-term relationship with previously faithful men.

316 In support of our hypothesis our findings in the PLC group demonstrate a clear

317 sex-dependent bias in mate choice with men expressing a greater interest than women in

318 having short-term relationships with previously unfaithful individuals. This therefore tends to

319 support proposed evolutionary sex-differences in mate choice priorities (Buss \& Schmitt,

320 1993; Oliver \& Hyde, 1993). In addition, we found some evidence to support previous

321 studies arguing that a sex difference in the perceived risk of infidelity is reflected in women

322 being more concerned by emotional infidelity but men by sexual infidelity (Buss, 2018; Buss

323 et al., 1992). Females more liked emotionally faithful males and males more liked sexually

324 faithful females. 
325 Again in support of our original hypothesis OXT administration increased

326 sex-differences in mate-choice priorities. Thus, in contrast to the PLC group, subjects in the

327 OXT group exhibited sex-differences in the influence that knowledge of previous fidelity or

328 infidelity had on attractiveness and likeability ratings and memory for prospective partners.

329 Importantly however, OXT administration had no effect on potential confounders such as

330 arousal and trustworthiness ratings and effects were also independent of relationship status.

331 More specifically, OXT increased men's attractiveness and likeability ratings of previously

332 unfaithful women but correspondingly decreased those for unfaithful men by women.

333 Furthermore, following OXT administration women found the face pictures of men

334 associated with previous infidelity less memorable, suggesting that they would be more likely

335 to only remember faithful individuals. Interestingly however, OXT did not alter the

336 sex-specific preferences for the attractiveness and likeability ratings given to individuals who

337 had previously exhibited emotional (female) as opposed to sexual (male) fidelity. This may

338 reflect the fact that the sex-dependent effects of OXT were mainly in the context of interest in

339 previous infidelity or that it may have less influence on such strongly established within-sex

340 patterns of preference. Both the sex-differences observed in the PLC group and in response to

341 OXT treatment were robust with all achieving medium or large effect sizes, thereby

342 confirming the appropriateness of the power analysis for the study.

343 While the sex-dependent effects of OXT on attraction and likeability ratings and

344 memory for faces occurred irrespective of relationship status, those for increasing interest in

345 having short or long-term relationships were restricted to single individuals. This finding

346 supports our hypothesis that OXT would enhance sex-dependent social and reproductive 
347 priorities (Gao et al., 2016; Hurlemann and Scheele, 2016) but particularly in single

348 individuals who have a higher priority for seeking a potential partner than those already in an

349 established relationship. That OXT primarily increased single men's interest in having

350 short-term relationships with women who had previously been unfaithful may reflect a high

351 priority for gaining sexual access to females. Similarly, single women's increased interest in

352 faithful males, and decreased interest in and memory for unfaithful ones, may reflect both a

353 higher priority for avoiding potential philandering males and preference for faithful

354 individuals who are more likely to provide a stable and secure relationship.

355 Oxytocin release associated with partner bonding across species is primarily evoked by 356 mating or sexual arousal as well as by affective touch (Hurlemann and Scheele, 2016; Li et

357 al., 2019), and can even occur in response to visual cues from the face (Fabre-Nys et al.,

358 1997). While there is some evidence that OXT can increase the perceived attractiveness of

359 the faces of unfamiliar members of the opposite sex (Hurlemann and Scheele, 2016) our

360 current findings emphasize that its release during initial social interactions might serve to

361 focus attention on pertinent information concerning a prospective partner's behavior and

362 history and not merely on their physical appearance. Indeed, previous studies have also

363 demonstrated that intranasal OXT administration can potently, and sex-dependently, alter

364 behavioral and neural responses to faces when they are paired with information on positive or

365 negative social qualities (Gao et al., 2016) and reduce recognition speed for positive romantic

366 and bonding-related words (Unkelbach et al., 2008). Thus, while OXT release can ultimately

367 promote the formation of partner bonds, it may first play a key role in highlighting the 
368 attractiveness of personal characteristics in a prospective partner which best match an

369 individual's current priorities.

370 The current study has several potential limitations. Firstly, the paradigm used of rating

371 attractiveness and mating preference for individuals based purely on their face pictures

372 associated with a verbal descriptor is commonly used, it is relatively artificial and it is

373 possible that results in contexts involving real social interactions might have been different.

374 Secondly, the subjects used in the study were primarily students and those in a relationship

375 had relatively short durations (>6 months). It is possible that both sex-differences in the PLC

376 group and the effects of OXT might have been influenced by age and also relationship

377 durations.

\section{Conclusions}

379 In summary, our findings demonstrate firstly that in the context of knowledge of a

380 prospective partner's fidelity or infidelity in previous relationships men who are either single

381 or in a relationship are more interested than women in having short-term relationships with

382 unfaithful individuals. Following OXT treatment this sex difference is both amplified and

383 extended such that men are even more interested in having short-term relationships with

384 unfaithful individuals and women even less so. Furthermore, women exhibit a greater interest

385 in faithful individuals than do men in the context of long-term relationships. In terms of mate

386 choice therefore, the sexes exhibit a differential interest in prospective partners who are

387 "stayers" or "strayers", and for single individuals with a current priority for finding a

388 prospective partner OXT release during romantic encounters may act to further widen this 
390 priorities before subsequently promoting romantic bonds with the most appropriate partners.

391

\section{Acknowledgements}

393 We thank Professor Trevor Robbins for valuable discussions and suggestions on the paper and

394 its findings. This project was supported by National Natural Science Foundation of Science

395 (NSFC) grant number 31530032.

396 Author Contributions

397 LX and KMK designed the experiment. LX, RL, XZ and WZ carried out the experiment. LX,

398 KMK, BB and QZ analyzed the experiment and LX, KMK and BB wrote the paper. All authors

399 contributed to the conception of the study and approved the paper.

400

401 Declaration of Interests

402 The authors declare that they have no competing interests.

403

404 References

405 Buss, D.M., 2018. Sexual and emotional infidelity: Evolved gender differences in jealousy

406 prove robust and replicable. Perspect. Psychol. Sci. 13, 155-160.

$407 \quad$ https://doi.org/10.1177/1745691617698225

408 Buss, D.M., Larsen, R.J., Westen, D., Semmelroth, J., 1992. Sex differences in jealousy:

409 Evolution, physiology, and psychology. Psychol. Sci. 3, 251-256.

$410 \quad$ https://doi.org/10.1111/j.1467-9280.1992.tb00038.x 
411 Buss, D.M., Schmitt, D.P., 1993. Sexual Strategies Theory: An evolutionary perspective on

412 human mating. Psychol. Rev. 100, 204-232.

$413 \quad$ https://doi.org/10.1037/0033-295X.100.2.204

414 Cavanaugh, J., Mustoe, A.C., Taylor, J.H., French, J.A., 2014. Oxytocin facilitates fidelity in

415 well-established marmoset pairs by reducing sociosexual behavior toward opposite-sex

416 strangers. Psychoneuroendocrinology 49, 1-10.

417 https://doi.org/10.1016/j.psyneuen.2014.06.020

418 Cohen, J., 1988. The Effect Size index: d, in: Statistical Power Analysis for the Behavioral

$419 \quad$ Sciences. pp. 20-26.

420 De Dreu, C.K.W., Kret, M.E., 2016. Oxytocin conditions intergroup relations through

421 upregulated in-group empathy, cooperation, conformity, and defense. Biol. Psychiatry

$422 \quad 79,165-173$. https://doi.org/10.1016/j.biopsych.2015.03.020

423 Donaldson, Z.R., Young, L.J., 2008. Oxytocin, Vasopressin, and the Neurogenetics of

424 Sociality. Science. 322, 900-904. https://doi.org/10.1126/science.1158668

425 Fabre $\square$ Nys, C., Ohkura, S., Kendrick, K.M., 1997. Male faces and odours evoke differential

426 patterns of neurochemical release in the mediobasal hypothalamus of the ewe during

427 oestrus: an insight into sexual motivation? Eur. J. Neurosci. 9, 1666-1677.

$428 \quad$ https://doi.org/10.1111/j.1460-9568.1997.tb01524.x

429 Fitzgerald, F.S., 1925. The Great Gatsby. Charles Scribner’s Sons, New York.

430 Gangestad, S.W., Haselton, M.G., Welling, L.L.M., Gildersleeve, K., Pillsworth, E.G.,

431 Burriss, R.P., Larson, C.M., Puts, D.A., 2016. How valid are assessments of conception

432 probability in ovulatory cycle research? Evaluations, recommendations, and theoretical 
433 implications. Evol. Hum. Behav. 37, 85-96.

434 https://doi.org/10.1016/j.evolhumbehav.2015.09.001

435 Gao, S., Becker, B., Luo, L., Geng, Y., Zhao, W., Yin, Y., Hu, J., Gao, Z., Gong, Q.,

436 Hurlemann, R., Yao, D., Kendrick, K.M., 2016. Oxytocin, the peptide that bonds the

437 sexes also divides them. Proc. Natl. Acad. Sci. U. S. A. 113, 7650-7654.

$438 \quad$ https://doi.org/10.1073/pnas.1602620113

439 Geng, Y., Zhao, W., Zhou, F., Ma, X., Yao, S., Hurlemann, R., Becker, B., Kendrick, K.M.,

440 2018. Oxytocin enhancement of emotional empathy: Generalization across cultures and

441 effects on amygdala activity. Front. Neurosci. 12, 512.

442 https://doi.org/10.3389/fnins.2018.00512

443 Guastella, A.J., Hickie, I.B., McGuinness, M.M., Otis, M., Woods, E.A., Disinger, H.M.,

444 Chan, H.K., Chen, T.F., Banati, R.B., 2013. Recommendations for the standardisation of

445 oxytocin nasal administration and guidelines for its reporting in human research.

446 Psychoneuroendocrinology 38, 612-625.

447 https://doi.org/10.1016/j.psyneuen.2012.11.019

448 Hu, J., Qi, S., Becker, B., Luo, L., Gao, S., Gong, Q., Hurlemann, R., Kendrick, K.M., 2015.

449 Oxytocin selectively facilitates learning with social feedback and increases activity and

450 functional connectivity in emotional memory and reward processing regions. Hum.

$451 \quad$ Brain Mapp. 36, 2132-2146. https://doi.org/10.1002/hbm.22760

452 Hurlemann, R., Scheele, D., 2016. Dissecting the role of oxytocin in the formation and loss of 453 social relationships. Biol. Psychiatry 79, 185-193.

$454 \quad$ https://doi.org/10.1016/j.biopsych.2015.05.013 
455 Kendrick, K.M., Guastella, A.J., Becker, B., 2017. Overview of human oxytocin research, in:

456 Hurlemann, R., Grinevich, V. (Eds.), Behavioral Pharmacology of Neuropeptides:

457 Oxytocin. Springer, Cham, pp. 321-348. https://doi.org/10.1007/7854_2017_19

458 Knopp, K., Scott, S., Ritchie, L., Rhoades, G.K., Markman, H.J., Stanley, S.M., 2017. Once a

459 Cheater, Always a Cheater? Serial Infidelity Across Subsequent Relationships. Arch.

460 Sex. Behav. 46, 2301-2311. https://doi.org/10.1007/s10508-017-1018-1

461 Lansford, J.E., 2009. Parental divorce and children's adjustment. Perspect. Psychol. Sci. 4,

$462 \quad$ 140-152. https://doi.org/10.1111/j.1745-6924.2009.01114.x

463 Lee, M.R., Scheidweiler, K.B., Diao, X.X., Akhlaghi, F., Cummins, A., Huestis, M.A.,

464 Leggio, L., Averbeck, B.B., 2018. Oxytocin by intranasal and intravenous routes reaches

465 the cerebrospinal fluid in rhesus macaques: determination using a novel oxytocin assay.

466 Mol. Psychiatry 23, 115. https://doi.org/10.1038/mp.2017.27

467 Li, Q., Becker, B., Wernicke, J., Chen, Y., Zhang, Y., Li, R., Le, J., Kou, J., Zhao, W.,

468 Kendrick, K.M., 2019. Foot massage evokes oxytocin release and activation of

469 orbitofrontal cortex and superior temporal sulcus. Psychoneuroendocrinology 101,

470 193-203. https://doi.org/https://doi.org/10.1016/j.psyneuen.2018.11.016

471 Luo, L., Becker, B., Geng, Y., Zhao, Z., Gao, S., Zhao, W., Yao, S., Zheng, X., Ma, X., Gao,

472 Z., Hu, J., Kendrick, K.M., 2017. Sex-dependent neural effect of oxytocin during

473 subliminal processing of negative emotion faces. Neuroimage 162, 127-137.

$474 \quad$ https://doi.org/10.1016/j.neuroimage.2017.08.079

475 Luo, R., Xu, L., Zhao, W., Ma, X., Xu, X., Kou, J., Gao, Z., Becker, B., Kendrick, K.M.,

476 2017. Oxytocin facilitation of acceptance of social advice is dependent upon the 
477 perceived trustworthiness of individual advisors. Psychoneuroendocrinology 83, 1-8.

$478 \quad$ https://doi.org/10.1016/j.psyneuen.2017.05.020

479 Oliver, B.M., Hyde, S.J., 1993. Gender Differences in Sexuality: A Meta- Analysis. Psychol.

480 Bull. 114, 29-51. https://doi.org/doi: 10.1037/0033-2909.114.1.29

481 Paloyelis, Y., Doyle, O.M., Zelaya, F.O., Maltezos, S., Williams, S.C., Fotopoulou, A.,

482 Howard, M.A., 2016. A spatiotemporal profile of in vivo cerebral blood flow changes

483 following intranasal oxytocin in humans. Biol. Psychiatry 79, 693-705.

$484 \quad$ https://doi.org/10.1016/j.biopsych.2014.10.005

485 Penton-Voak, I.S., Perrett, D.I., Castles, D.L., Kobayashi, T., Burt, D.M., Murray, L.K.,

486 Minamisawa, R., 1999. Menstrual cycle alters face preference. Nature 399, 741.

$487 \quad$ https://doi.org/10.1038/21557

488 Place, S.S., Todd, P.M., Penke, L., Asendorpf, J.B., 2010. Humans show mate copying after

489 observing real mate choices. Evol. Hum. Behav. 31, 320-325.

$490 \quad$ https://doi.org/10.1016/j.evolhumbehav.2010.02.001

491 Preckel, K., Scheele, D., Kendrick, K.M., Maier, W., Hurlemann, R., 2014. Oxytocin

492 facilitates social approach behavior in women. Front. Behav. Neurosci. 8, 191.

493 https://doi.org/10.3389/fnbeh.2014.00191

494 Quintana, D.S., Westlye, L.T., Alnæs, D., Rustan, Ø.G., Kaufmann, T., Smerud, K.T.,

495 Mahmoud, R.A., Djupesland, P.G., Andreassen, O.A., 2016. Low dose intranasal

496 oxytocin delivered with Breath Powered device dampens amygdala response to

497 emotional stimuli: A peripheral effect-controlled within-subjects randomized

498 dose-response fMRI trial. Psychoneuroendocrinology 69, 180-188. 
500 Quintana, D.S., Westlye, L.T., Smerud, K.T., Mahmoud, R.A., Andreassen, O.A., Djupesland,

501 P.G., 2018. Saliva oxytocin measures do not reflect peripheral plasma concentrations

502 after intranasal oxytocin administration in men. Horm. Behav. 102, 85-92.

$503 \quad$ https://doi.org/10.1016/j.yhbeh.2018.05.004

504 Scheele, D., Striepens, N., Gunturkun, O., Deutschlander, S., Maier, W., Kendrick, K.M.,

505 Hurlemann, R., 2012. Oxytocin modulates social distance between males and females. J.

$506 \quad$ Neurosci. 32, 16074-16079. https://doi.org/10.1523/JNEUROSCI.2755-12.2012

507 Scheele, D., Striepens, N., Kendrick, K.M., Schwering, C., Noelle, J., Wille, A., Schläpfer,

508 T.E., Maier, W., Hurlemann, R., 2014. Opposing effects of oxytocin on moral judgment

509 in males and females. Hum. Brain Mapp. 35, 6067-6076.

$510 \quad$ https://doi.org/10.1002/hbm.22605

511 Scheele, D., Wille, A., Kendrick, K.M., Stoffel-Wagner, B., Becker, B., Güntürkün, O.,

512 Maier, W., Hurlemann, R., 2013. Oxytocin enhances brain reward system responses in

513 men viewing the face of their female partner. Proc. Natl. Acad. Sci. U. S. A. 110,

514 20308-20313. https://doi.org/10.1073/pnas.1314190110

515 Shin, N.Y., Park, H.Y., Jung, W.H., Kwon, J.S., 2018. Effects of Intranasal Oxytocin on

516 Emotion Recognition in Korean Male: A Dose-Response Study. Psychiatry Investig. 15,

517 710. https://doi.org/10.30773/pi.2018.02.19

518 Spengler, F.B., Schultz, J., Scheele, D., Essel, M., Maier, W., Heinrichs, M., Hurlemann, R.,

519 2017. Kinetics and dose dependency of intranasal oxytocin effects on amygdala

520 reactivity. Biol. Psychiatry 82, 885-894. https://doi.org/10.1016/j.biopsych.2017.04.015 
521 Striepens, N., Kendrick, K.M., Hanking, V., Landgraf, R., Wüllner, U., Maier, W.,

522 Hurlemann, R., 2013. Elevated cerebrospinal fluid and blood concentrations of oxytocin

523 following its intranasal administration in humans. Sci. Rep. 3, 3440.

524 https://doi.org/10.1038/srep03440

525 Takahashi, H., Matsuura, M., Yahata, N., Koeda, M., Suhara, T., Okubo, Y., 2006. Men and

526 women show distinct brain activations during imagery of sexual and emotional infidelity.

527 Neuroimage 32, 1299-1307. https://doi.org/10.1016/j.neuroimage.2006.05.049

528 Unkelbach, C., Guastella, A.J., Forgas, J.P., 2008. Oxytocin selectively facilitates recognition

529 of positive sex and relationship words. Psychol. Sci. 19, 1092-1094.

$530 \quad$ https://doi.org/10.1111/j.1467-9280.2008.02206.x

531 Xu, L., Ma, X., Zhao, W., Luo, L., Yao, S., Kendrick, K.M., 2015. Oxytocin enhances

532 attentional bias for neutral and positive expression faces in individuals with higher

$533 \quad$ autistic traits. Psychoneuroendocrinology 62, 352-358.

534 https://doi.org/10.1016/j.psyneuen.2015.09.002

535 Zhao, W., Geng, Y., Luo, L., Zhao, Z., Ma, X., Xu, L., Yao, S., Kendrick, K.M., 2017.

536 Oxytocin increases the perceived value of both self-and other-owned items and alters

537 medial prefrontal cortex activity in an endowment task. Front. Hum. Neurosci. 11, 272.

$538 \quad$ https://doi.org/10.3389/fnhum.2017.00272

539 Zhao, W., Ma, X., Le, J., Ling, A., Xin, F., Kou, J., Zhang, Y., Luo, R., Becker, B., Kendrick,

$540 \quad$ K.M., 2018. Oxytocin biases men to be more or less tolerant of others' dislike dependent

$541 \quad$ upon their relationship status. Psychoneuroendocrinology 88, 167-172.

$542 \quad$ https://doi.org/10.1016/j.psyneuen.2017.12.010 
bioRxiv preprint doi: https://doi.org/10.1101/416198; this version posted June 14,2019 . The copyright holder for this preprint (which was not certified by peer review) is the author/funder, who has granted bioRxiv a license to display the preprint in perpetuity. It is made available under aCC-BY-NC 4.0 International license. 


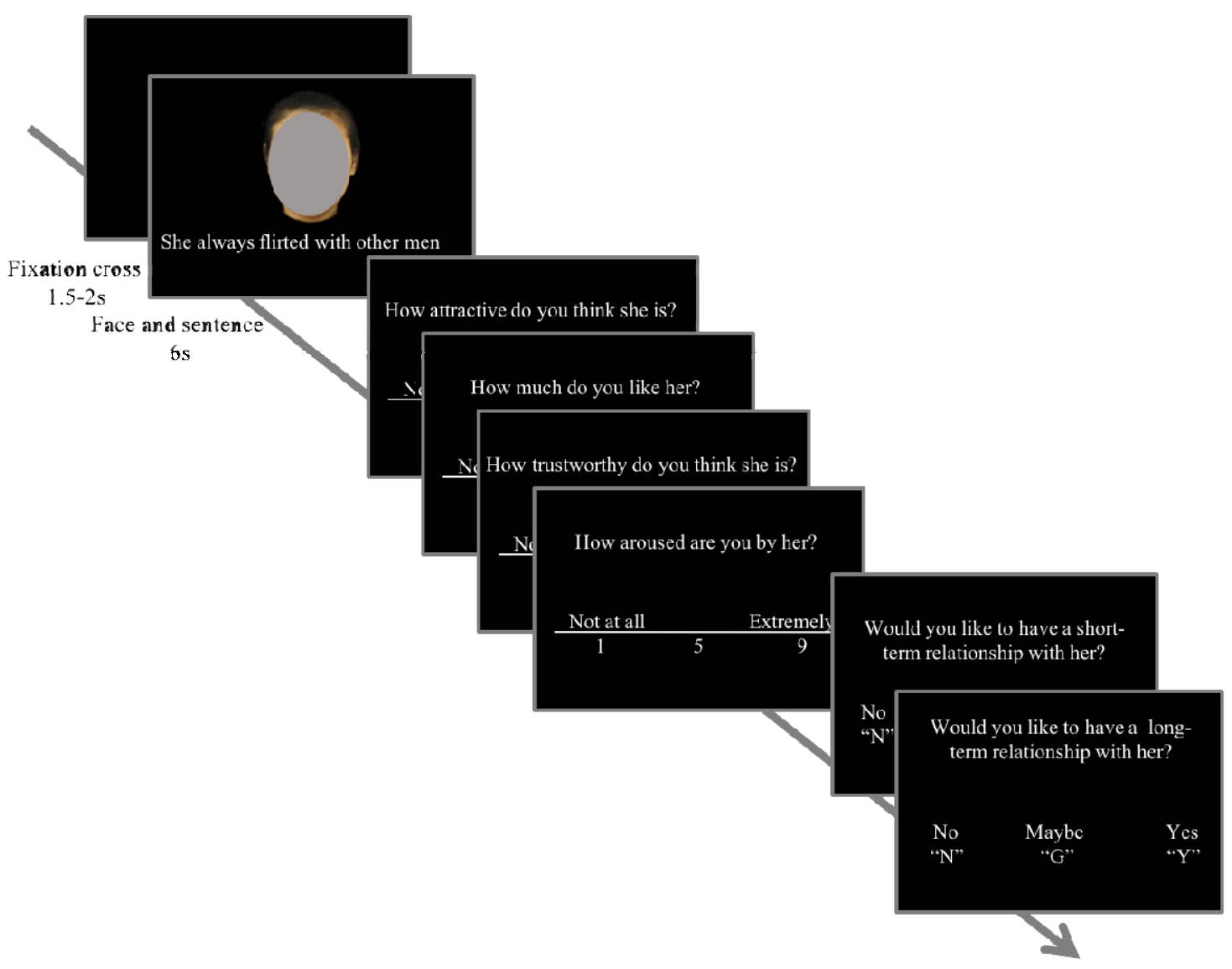

545 Fig. 1. Example of a single trial in the rating task. Following a 1.5 2 second fixation cross,

546 each facial picture (unknown, opposite sex) was shown for 6 seconds and paired with a

547 sentence describing a behavior indicative of fidelity or infidelity (either emotional or sexual)

548 he/she exhibited during a previous relationship. Each subject viewed 10 trials for each

549 fidelity/infidelity type - emotional fidelity, sexual fidelity, emotional infidelity and sexual

550 infidelity. 


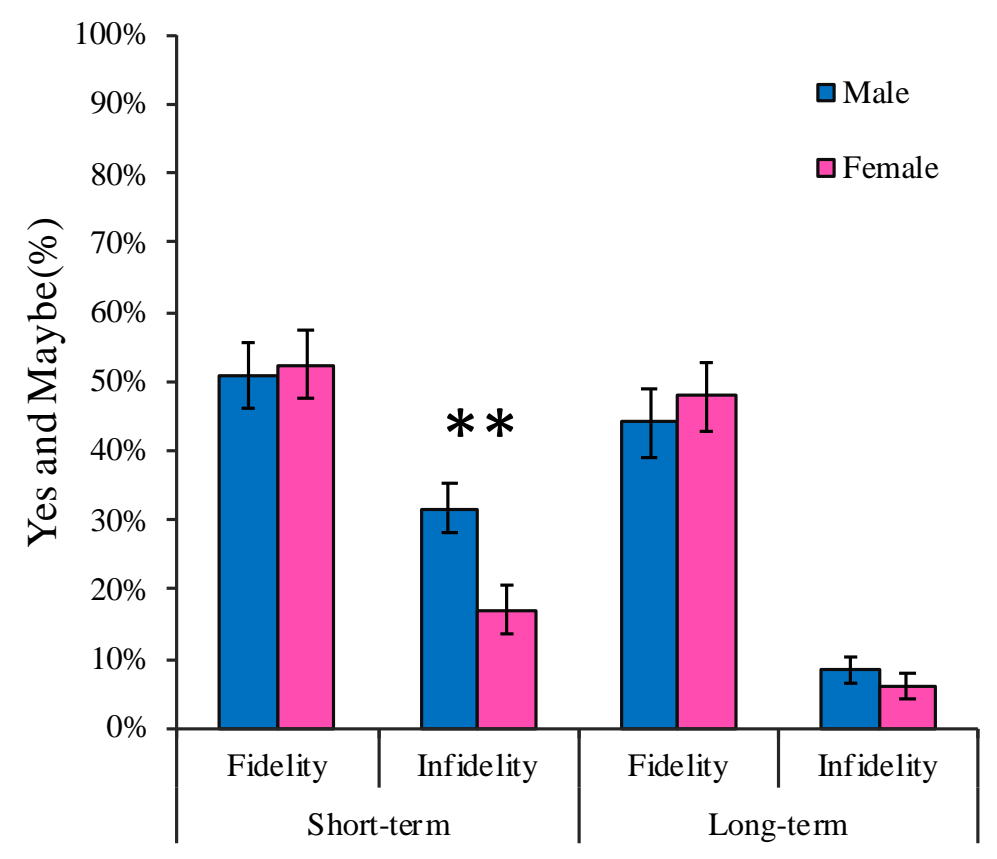

552 Fig. 2. Sex difference in the percentage of yes/maybe responses made by subjects for having

553 a short-term, but not long-term, relationship with individuals showing previous infidelity in

554 the placebo treated group. Data from single individuals and those in a relationship are

555 combined. Bars represent means and standard errors. ${ }^{*} p<0.01$ for males vs. females. 

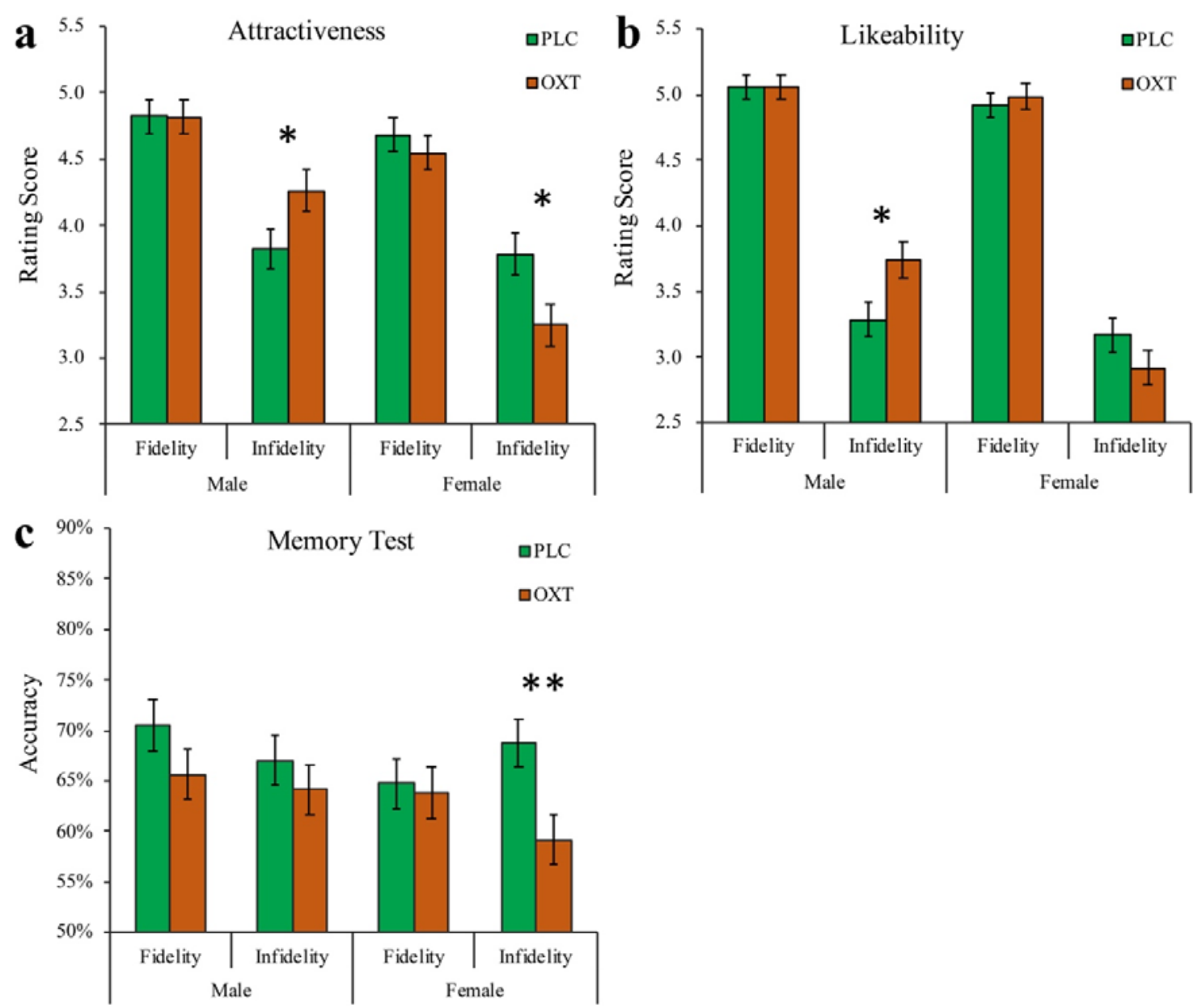

558 Fig. 3. Effects of oxytocin (OXT) on attractiveness (a) likeability (b) and recognition

559 memory (c) for faces of the opposite sex associated with previous fidelity or infidelity in all

560 male and female subjects. Bars represent means and standard errors. ${ }^{*} p<0.05, * * p<0.01$

561 OXT vs. placebo (PLC). 

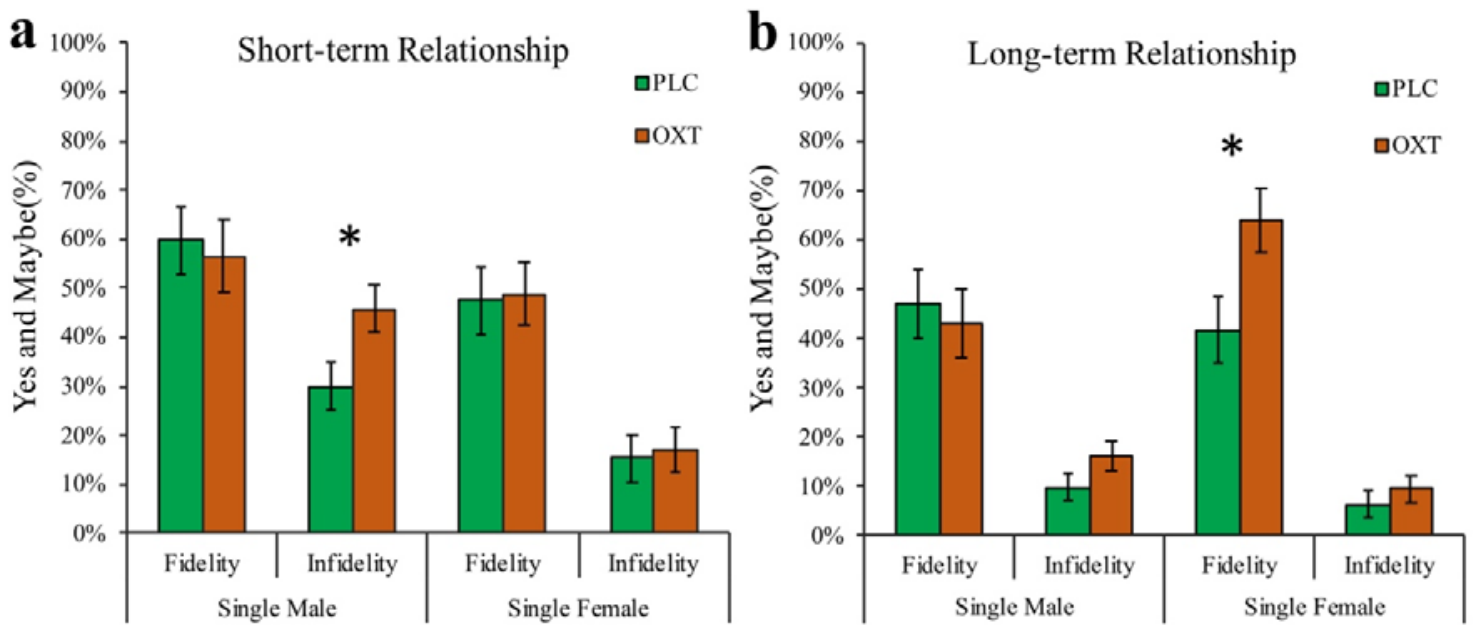

564 Fig. 4. Effect of oxytocin (OXT) on percentage of yes/maybe responses in single male and

565 female subjects for having a short-term (a) or long-term relationship (b) with an individual of

566 the opposite sex associated with previous fidelity or infidelity. Bars represent means and

567 standard errors. ${ }^{*} p<0.05$ OXT vs. placebo (PLC). 

aCC-BY-NC 4.0 International license.

569 Table 1. Ages and questionnaire scores in the four experimental groups (mean \pm S.E.M.)

\begin{tabular}{|c|c|c|c|c|c|}
\hline \multirow{2}{*}{ Measurements } & \multicolumn{2}{|c|}{ Placebo } & \multicolumn{2}{|c|}{ Oxytocin } & \multirow{2}{*}{$\begin{array}{c}\text { Sex } x \text { Treatment } \\
p \text {-value }\end{array}$} \\
\hline & Male & Female & Male & Female & \\
\hline Age(years) & $23.0 \pm 0.3$ & $22.8 \pm 0.3$ & $22.9 \pm 0.3$ & $22.7 \pm 0.3$ & 0.872 \\
\hline Positive and Negative Affective Scale (PANAS) -Positive & $31.6 \pm 0.8$ & $29.2 \pm 0.9$ & $28.8 \pm 0.9$ & $28.5 \pm 1.0$ & 0.237 \\
\hline Positive and Negative Affective Scale (PANAS) -Negative & $21.7 \pm 1.3$ & $18.1 \pm 1.1$ & $19.2 \pm 1.1$ & $18.0 \pm 1.0$ & 0.306 \\
\hline NEO-Five Factor Inventory-Agreeableness & $42.4 \pm 0.7$ & $41.4 \pm 0.6$ & $40.7 \pm 0.6$ & $40.9 \pm 0.8$ & 0.365 \\
\hline NEO-Five Factor Inventory-Conscientiousness & $42.7 \pm 0.8$ & $41.7 \pm 0.7$ & $41.4 \pm 0.8$ & $42.3 \pm 0.8$ & 0.220 \\
\hline NEO-Five Factor Inventory-Extraversion & $41.2 \pm 0.8$ & $38.8 \pm 1.0$ & $39.9 \pm 1.0$ & $40.8 \pm 0.8$ & 0.070 \\
\hline NEO-Five Factor Inventory-Neuroticism & $33.8 \pm 1.3$ & $34.5 \pm 1.1$ & $34.4 \pm 1.1$ & $34.1 \pm 1.2$ & 0.667 \\
\hline NEO-Five Factor Inventory-Openness & $40.4 \pm 0.7$ & $38.2 \pm 0.9$ & $39.8 \pm 0.8$ & $39.3 \pm 0.8$ & 0.275 \\
\hline Self-Esteem Scale (SES) & $30.6 \pm 0.7$ & $31.3 \pm 0.6$ & $30.5 \pm 0.6$ & $30.8 \pm 0.8$ & 0.811 \\
\hline Interpersonal Reactivity Index (IRI) & $50.0 \pm 1.6$ & $51.9 \pm 1.5$ & $45.0 \pm 1.4$ & $50.8 \pm 1.6$ & 0.200 \\
\hline Autism Spectrum Quotient (ASQ) & $20.0 \pm 0.7$ & $20.7 \pm 0.9$ & $20.7 \pm 0.6$ & $19.7 \pm 0.8$ & 0.254 \\
\hline Beck Depression Inventory (BDI-II) & $8.1 \pm 0.9$ & $7.9 \pm 1.2$ & $8.8 \pm 1.3$ & $7.2 \pm 0.9$ & 0.512 \\
\hline Liebowitz's Social Anxiety Scale (LSAS)-Avoid & $20.8 \pm 1.9$ & $19.1 \pm 1.8$ & $20.2 \pm 1.4$ & $21.5 \pm 1.9$ & 0.421 \\
\hline Liebowitz's Social Anxiety Scale (LSAS)-Fear & $24.7 \pm 2.0$ & $21.6 \pm 1.6$ & $22.2 \pm 1.5$ & $25.3 \pm 2.1$ & 0.089 \\
\hline Passionate Love Scale (PLS) & $103.4 \pm 2.4$ & $99.0 \pm 2.3$ & $97.8 \pm 2.0$ & $96.7 \pm 2.5$ & 0.476 \\
\hline Love Attitude Scale (LAS)-Agape & $26.9 \pm 0.6$ & $22.0 \pm 0.5$ & $25.2 \pm 0.6$ & $20.8 \pm 0.5$ & 0.686 \\
\hline Love Attitude Scale (LAS)-Eros & $24.1 \pm 0.5$ & $23.5 \pm 0.5$ & $23.4 \pm 0.6$ & $23.6 \pm 0.6$ & 0.529 \\
\hline Love Attitude Scale (LAS)-Ludus & $19.3 \pm 0.7$ & $19.0 \pm 0.6$ & $19.9 \pm 0.5$ & $18.9 \pm 0.5$ & 0.573 \\
\hline Love Attitude Scale (LAS)-Mania & $21.2 \pm 0.7$ & $19.7 \pm 0.7$ & $21.0 \pm 0.6$ & $19.9 \pm 0.7$ & 0.706 \\
\hline Love Attitude Scale (LAS)-Pragma & $22.6 \pm 0.7$ & $23.1 \pm 0.6$ & $20.9 \pm 0.7$ & $23.0 \pm 0.6$ & 0.213 \\
\hline Love Attitude Scale (LAS)-Storge & $22.8 \pm 0.7$ & $21.5 \pm 0.7$ & $21.3 \pm 0.7$ & $21.7 \pm 0.8$ & 0.261 \\
\hline General Trust Scale (GTS) & $32.0 \pm 0.5$ & $31.6 \pm 0.6$ & $31.2 \pm 0.6$ & $31.8 \pm 0.7$ & 0.380 \\
\hline Tendency to Forgive Scale (TTF) & $14.3 \pm 0.5$ & $14.3 \pm 0.5$ & $14.1 \pm 0.6$ & $13.9 \pm 0.7$ & 0.846 \\
\hline Attitudes toward Forgiveness Scale (ATF) & $28.5 \pm 0.7$ & $27.9 \pm 0.7$ & $27.4 \pm 0.6$ & $26.5 \pm 0.7$ & 0.808 \\
\hline Trait Forgivingness Scale (TFS) & $32.7 \pm 0.9$ & $32.5 \pm 0.9$ & $32.0 \pm 0.8$ & $30.9 \pm 1.0$ & 0.614 \\
\hline
\end{tabular}




\section{Supporting Information}

\section{$573 \quad$ Oxytocin amplifies evolutionary sex differences in human mate choice}

574 Lei Xu, Benjamin Becker, Ruixue Luo, Xiaoxiao Zheng, Weihua Zhao, Qiong Zhang* and

575 Keith M. Kendrick ${ }^{*}$

576

577 The Clinical Hospital of Chengdu Brain Science, MOE Key Laboratory for NeuroInformation,

578 University of Electronic Science and Technology of China, Chengdu 611731, China.

\section{Supplementary Methods}

580 To control for potential confounds, before intranasal treatment all subjects completed a range

581 of validated questionnaires (Chinese versions) measuring mood, personality traits and

582 attitudes toward love, trust and forgiveness. These included: Positive and Negative Affective

583 Schedule - PANAS (Watson et al., 1988); NEO-Five Factor Inventory - NEO-FFI (Costa

584 and Mccrae, 1989); Self-Esteem Scale - SES (Rosenberg, 1965); Interpersonal Reactivity

585 Index - IRI (Davis, 1980); Autism Spectrum Quotient - ASQ (Baron-Cohen et al., 2001);

586 Beck's Depression Inventory - BDI (Beck et al., 1996); Leibowitz's Social Anxiety Scale -

587 LSAS (Liebowitz, 1987); Passionate Love Scale - PLS (Hatfiled and Sprecher, 1986); Love

588 Attitude Scale - LAS (Hendrick and Hendrick, 1986); General Trust Scale - GTS (Siegrist et

589 al., 2005); Tendency to Forgive Scale - TTF (Brown, 2003); Attitudes toward Forgiveness

590 Scale - ATF (Brown, 2003); Trait Forgivingness Scale - TFS (Berry et al., 2005). Univariate

591 ANOVAs on questionnaires and age showed no significant differences between the OXT-

592 and PLC-treated males and females (sex x treatment interaction: all ps >0.070; See Table 1). 


\section{Supplementary Results}

594 Repeated-measures ANOVAs added menstrual cycle as a between-subject factor in

595 female subjects suggested that the stage of their menstrual cycle did not influence our

596 findings. There were no significant interactions of menstrual cycle and fidelity or interactions

597 of menstrual cycle and type for rating scores, memory and short-term or long-term

598 relationship preferences in the PLC group (all $p s>0.100$ ). For the effects of OXT there were

599 also no significant interactions of menstrual cycle, treatment and fidelity for rating scores,

600 memory and short-term or long-term relationship preferences (all $p s>0.390$ ).

601

602 References

603 Baron-Cohen, S., Wheelwright, S., Skinner, R., Martin, J., Clubley, E., 2001. The autism, malesand females, scientists and mathematicians. J. Autism Dev. Disord. 31,

607 Beck, A.T., Steer, R.A., Brown, G.K., 1996. Beck depression inventory-II. The Psychological

608 Corporation, San Antonio, TX.

609 Berry, J.W., Worthington, E.L., O’Connor, L.E., Parrott, L., Wade, N.G., 2005.

610 Forgivingness, vengeful rumination, and affective traits. J. Pers. 73, 183-226.

$611 \quad$ https://doi.org/10.1111/j.1467-6494.2004.00308.x

612 Brown, R.P., 2003. Measuring Individual Differences in the Tendency to Forgive: Construct

613 Validity and Links with Depression Measuring Individual Differences in the Tendency

614 to Forgive: Construct Validity and Links With Depression. Personal. Soc. Psychol. Bull. 
$615 \quad 29,759-771$. https://doi.org/10.1177/0146167203029006008

616 Costa, P., Mccrae, R.R., 1989. The NEO-PI/NEO-FFI manual supplement. Psychological

617 Assessment Resources, Odessa, FL.

618 Davis, M.H., 1980. A multidimensional approach to individual differences in empahty. Cat.

619 Sel. Doc. Psychol. 40, 3480.

620 Hatfiled, E., Sprecher, S., 1986. Measuring passionate love in intimate relations. J. Adolesc. 9, 383-410. https://doi.org/10.1016/S0140-1971(86)80043-4

622 Hendrick, C., Hendrick, S., 1986. A theory and method of love. J. Pers. Soc. Psychol. 50,

623 392-402. https://doi.org/10.1037/0022-3514.50.2.392

624 Liebowitz, M.R., 1987. Social phobia, in: Anxiety. Karger Publishers, pp. 141-173.

625 https://doi.org/10.1159/000414022

626 Rosenberg, M., 1965. Society and the adolescent self-image. Princeton university press

627 Princeton, NJ.

628 Siegrist, M., Keller, C., Barle, T.C., Gutscher, H., 2005. Effects of general trust on

629 cooperation in the investment game and in a social dilemma Unpublished manuscript,

$630 \quad$ Institute for Environmenta.

631 Watson, D., Clark, L.A., Tellegen, A., 1988. Development and validation of brief measures

632 of positive and negative affect: the PANAS scales. J. Pers. Soc. Psychol. 54, 1063-1070.

$633 \quad$ https://doi.org/10.1037/0022-3514.54.6.1063

635 Table S1. Examples of sentences describing sexual and emotional fidelity or infidelity

\begin{tabular}{ll}
\hline Type & Sentence Examples \\
\hline Emotional Fidelity & He/She always ignored other women/men who tried to flirt with him/her. \\
\hline
\end{tabular}


He/She always refused to go out on a date with other women/men.

Sexual Fidelity He/She threw wine on his/her female/male client's face when she/he tried to seduce him/her.

$\mathrm{He} / \mathrm{She}$ refused to have sex with his/her boss even though that would have resulted in gaining a promotion.

Emotional Infidelity He/She expressed his/her love to another woman/man without his/her girlfriend/boyfriend knowing.

$\mathrm{He} / \mathrm{She}$ sent many romantic text messages to another woman/man.

Sexual Infidelity $\quad$ He/She had sex with girlfriend's/boyfriend's best friend.

$\mathrm{He} / \mathrm{She}$ gave another woman/man oral sex.
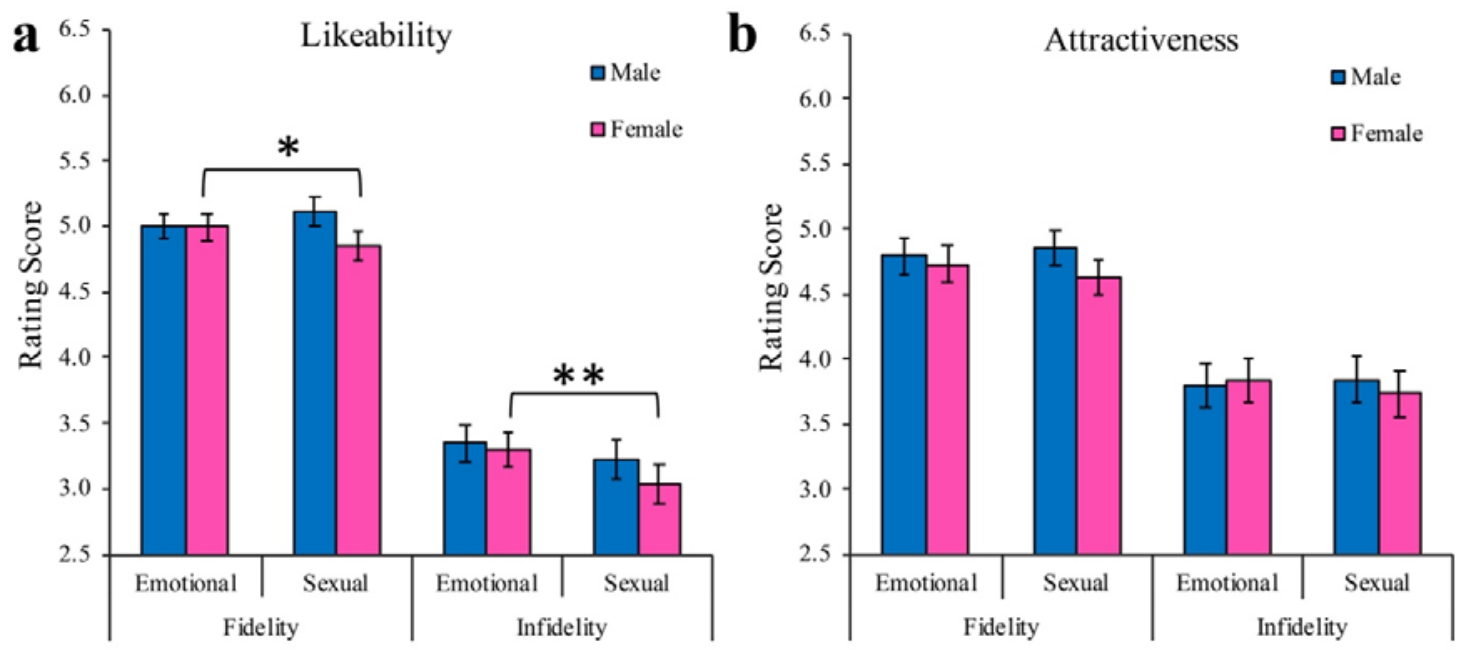

639 Fig. S1. Sex difference in likeability and attractiveness ratings in the placebo (PLC) treated

group. $* p<0.05, * * p<0.01$. 